\title{
VISUALIZAÇÃO DE INTEGRAIS DEPENDENTES DE PARÂMETROS COM ARRIMO NO SOFTWARE GEOGEBRA: UMA ENGENHARIA DIDÁTICA PARA SEU ENSINO
}

\author{
Maria Vanísia Mendonça de Lima ${ }^{1}$, Francisco Regis Vieira Alves ${ }^{2}$ \\ Instituto Federal de Educação, Ciência e Tecnologia do Ceará \\ ${ }^{1}$ Campus de Cedro \\ ${ }^{2}$ Programa de Pós-graduação em Ensino de Ciências e Matemática, campus de Fortaleza \\ <vanisialima@bol.com.br>.<fregis@ifce.edu.br>
}

DOI: $10.21439 /$ conexoes.v9i4.924

\begin{abstract}
Resumo. Para este trabalho, o objetivo é apresentar os resultados parciais de uma pesquisa em andamento para o Programa de Mestrado Acadêmico em Ensino de Ciências e Matemática, do Instituto Federal de Educação, Ciência e Tecnologia do Estado do Ceará - IFCE. Dessa forma, de modo preliminar, apresenta-se uma revisão da literatura com o tema integrais impróprias ou generalizadas. O referido conceito permite a descrição do modelo de integrais dependentes de parâmetros, que constituem sua generalização. Logo em seguida, discute-se a perspectiva de ensino das mesmas, segundo Dana-Picard, que leva em consideração a exploração da tecnologia, do contexto histórico, bem como as relações conceituais que compõem uma vizinhança cognitiva. Por fim, alguns exemplos específicos são significados com origem no uso do Software GeoGebra, tendo em vista a interpretação qualitativa do processo matemático em questão, com arrimo na visualização.
\end{abstract}

Palavras-chaves: Integrais Impróprias. Integrais dependentes de parâmetros. Visualização. Software Geogebra. Ensino.

\begin{abstract}
For this work, the aim is to present some preliminary results of an in progress study for the Academic Master's Program in Science and Mathematics Teaching of the Federal Institute of Education, Science and Technology of State of Ceara - IFCE. Thus, in a preliminary way, we present a literature review with a theme related to the concept improper or generalized integrals. Such concept allows the description of the another model, named dependent parameters integrals, which constitutes it's generalization. Soon after, we discussed a teaching perspective according to Dana-Picard, which takes into account the exploitation of Technology, the historical context and the conceptual relationships that comprise a cognitive neighborhood. Finally, some specific examples are made from the use of GeoGebra Software, in other a qualitative interpretation related a mathematical process concerned with the explore of the visualization's help.
\end{abstract}

Keywords: Improper Integrals. Visualization. Software GeoGebra. Teaching.

\section{INTRODUÇÃO E BREVE REVISÃO DA LITE- RATURA}

A noção de integral imprópria ou generalizada constitui noção unificadora e generalizadora relativamente ao processo de integração, segundo Riemann. A referida noção permite pensarmos a determinação de áreas sobre curvas, não apenas para funções contínuas no espaço bidimensional, mas para uma classe mais geral de funções.
Por outro lado, no contexto do ensino do Cálculo Integral, registramos enorme profusão de trabalhos relativos à noção de integral definida (BERMÚDEZ, 2011; HSIA, 2006; MILOVANOVIĆ; TAKAČI; MILAJIĆ, 2011; SEVIMLI; DELICE. 2011, SEVIMLI; DELICE, 2012; VANINSKY, 2015). No que concerne a produção de investigações que se detêm ao ensino/aprendizagem de integrais generalizadas ou integrais impróprias, de- 
VISUALIZAÇÃO DE INTEGRAIS DEPENDENTES DE PARÂMETROS COM ARRIMO NO SOFTWARE GEOGEBRA: UMA ENGENHARIA DIDÁTICA PARA SEU ENSINO

paramos uma produção representativa apenas em outros países (GONZÁLEZ-MARTÍN; CAMACHO 2004; GONZÁLEZ-MARTÍN; MACHÍN 2005; GONZÁLEZ-MARTÍN, 2005a, GONZÁLEZMARTÍN 2005b, TOMÉ, 2011). Por outro lado, registramos ainda outros escritos científicos que discutem o papel da visualização e do uso de alguns softwares específicos no ensino de integrais (ALVES. 2012; ALVES; LOPES, 2013; ALVES, 2014a; ALVES 2014b).

Em sua tese de doutorado, Bermúdez (2011) investigou o problema da compreensão do conceito de integral definida, no contexto de estudantes universitários, no âmbito de um curso de Engenharia. Seu estudo se enquadra no campo da Didática de Matemática e do Pensamento Matemático Avançado. Bermúdez (2011. p. 20)(tradução nossa) assinala a relevância curricular do seu objeto matemático, quando observa que "o conceito de integral definida é um dos conceitos fundamentais em Análise, se inclui no currículo de diversas carreiras e, de modo concreto, aparece nos planos de estudo da graduação em Matemática na Colômbia”. Bermudéz desenvolveu uma investigação particular relativa à noção de integral definida. Depreendemos, tal fato, a partir de sua declaração:

\footnotetext{
O conceito de integral definida, de acordo com nossa experiência e os resultados obtidos em diversas investigações, apresenta dificuldades para os estudantes que se manifestam mediante uma utilização mecânica, algorítmica e memoristica de sua definição. Não conseguem êxito em estabelecer uma conexão entre o pensamento numérico, algébrico e analítico. Manifestam problemas para interpretar gráficos abaixo de curvas, quando passa de ser positivo para negativo ou manifesta descontinuidades. Em outros casos, pensam a integral somente associada ao conceito de área, todavia, dissociada de outros contextos. Demonstram ainda dificuldades para aplicar as propriedades da integral definida BERMÚDEZ 2011 p. 20)(tradução nossa).
}

Um pouco mais adiante, Bermúdez (2011, p. 20 21) acentua os seguintes problemas manifestados pelos alunos na aprendizagem de integral definida, a saber: (i) geralmente os estudantes identificam integral com primitiva. A integral, para eles, não envolve nenhum processo de convergência, nem tampouco um aspecto geométrico. É, portanto, um processo eminentemente algébrico. São capazes de aplicar a integral, ignorando por completo as respectivas somas de Riemann; (ii) os estudantes geralmente identificam as integrais definidas com a regra de Barrow, inclusive quando não pode aplicar-se; (iii) falta de conexão e link conceitual en- tre a noção de integral definida e o conceito de área, pois, não conseguem estabelecer relação entre uma representação gráfica e uma representação analítica; (iv) o predomínio/predileção do modo de resolução algébrico sobre o gráfico.

Na Figura 1, Bermúdez (2011) analisa a produção de mapas conceituais e sua aplicação na resolução de tarefas. Este autor, indica ainda em suas considerações finais, que: os alunos associam a noção de integral definida com um algoritmo para o cálculo, desconsiderando as condições para sua aplicação; afirmam que a integral definida permite, apenas, a aproximação do valor de área; afirmam que continuidade implica em derivabilidade e extraem tal propriedade errônea para o contexto de integrabilidade.

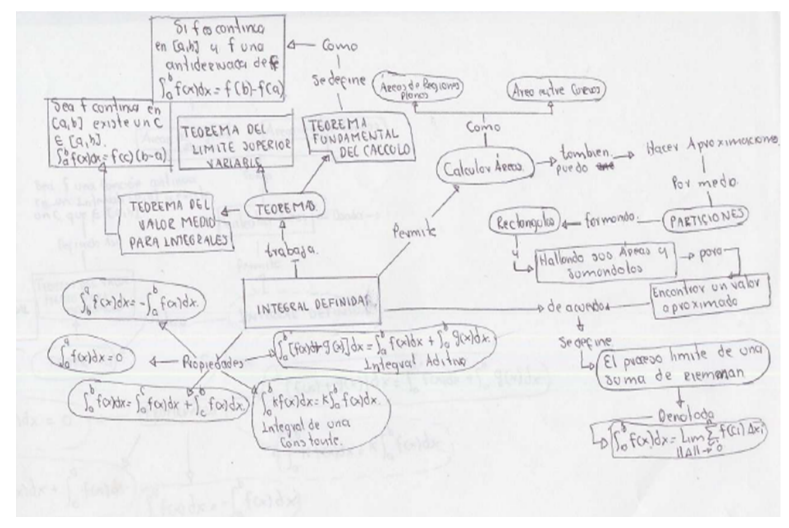

Figura 1: Imagem da análise da produção de mapas conceituais relativos ao uso das integrais definidas e propriedades na resolução de determinadas tarefas. Fonte: Bermudéz (2011, p. 199).

Tomé (2011) em sua tese intitulada Integral Definida, Cálculo Mental e Novas Tecnologias, desenvolveu um estudo experimental, no contexto de cursos de bacharelado. No rol de suas considerações finais, assinalamos uma relativa à seguinte conclusão: "os alunos compreendem e aprendem melhor a integral definida na medida em que se constrói uma sequência adequada de ensino" (TOMÉ, 2011, p. 650, tradução nossa).

A descrição da sequência de ensino de Tomé envolveu o uso de softwares de Matemática. E, tanto no contexto de resolução, como no contexto de uso de alguns softwares em sala de aula, Tomé (2011, p. 652, tradução nossa) registrou que "os alunos aplicam pseudopropriedades ao cálculo mental de primitivas elementares". Em relação a tal fato, o autor registrou estratégias recorrentes envolvendo erros recorrentes.

Cabe observar que, apesar de fornecer determinados elementos que podem/tendem a se manifestar em outro contexto de aprendizagem, os estudos anteriores 
VISUALIZAÇÃO DE INTEGRAIS DEPENDENTES DE PARÂMETROS COM ARRIMO NO SOFTWARE GEOGEBRA: UMA ENGENHARIA DIDÁTICA PARA SEU ENSINO

dizem respeito ao conceito de integral definida. Doravante, buscamos discutir o relato de trabalhos relacionados com integrais generalizadas ou integrais impróprias.

Para tanto, encontramos o estudo desenvolvido por González-Martín e Camacho (2004) que analisaram as respostas de um grupo de alunos de Matemática, no primeiro ano de universidade. O questionário aplicado por esses autores versava sobre situações problema envolvendo a noção de integral imprópria. Na Figura 2 González-Martín e Camacho (2004, p. 77) comentam o caso de um aluno que produziu um desenho, que expressa a ideia de função, representada por uma curva, sem patologias, num intervalo fechado. Ao lado esquerdo, os autores comentam a descrição de retângulos no intervalo considerado.
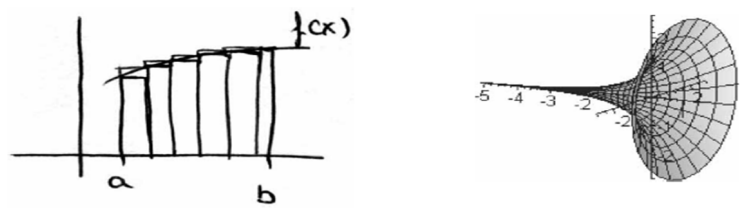

Figura 2: Desenhos relacionados com a noção de integral imprópria. Fonte: González-Martín e Camacho (2004).

Por outro lado, ainda no que concerne a Figura 2 González-Martín e Camacho (2004, p. 87) apresentam uma situação envolvendo a Trombeta do Anjo Gabriel. Do ponto de vista matemático, sabemos que a superfície gerada pela rotação da função, possui comprimento infinito. Por outro lado, por intermédio do processo de integração, vemos que o volume, delimitado pela superfície, na figura 2, é finito. A partir desses fatos, podemos explorar uma situação didática com os estudantes, com o intuito de explicar-lhes que, embora possamos encher toda a Trombeta, com um volume finito de tinta, nunca poderemos pintá-la em todo seu comprimento, posto que a mesma é infinita (ver Figura 2, ao lado direito).

Em outra investigação, González-Martín (2005b) apresentam o relato de um estudo envolvendo um grupo de alunos, no primeiro ano de universidade, com o objetivo de identificar as dificuldades e erros, registrados no primeiro ano de licenciatura, no que concerne ao conceito de integral imprópria.

González-Martín (2005b p. 81) comentam que "um dos principais problemas reside no entendimento da convergência destas integrais. No ensino universitário atual, se centra na atenção e memorização de critérios e múltiplos exercícios; embora, quase não se interpreta os resultados obtidos". Pouco mais adiante, alertam para o seguinte fato:

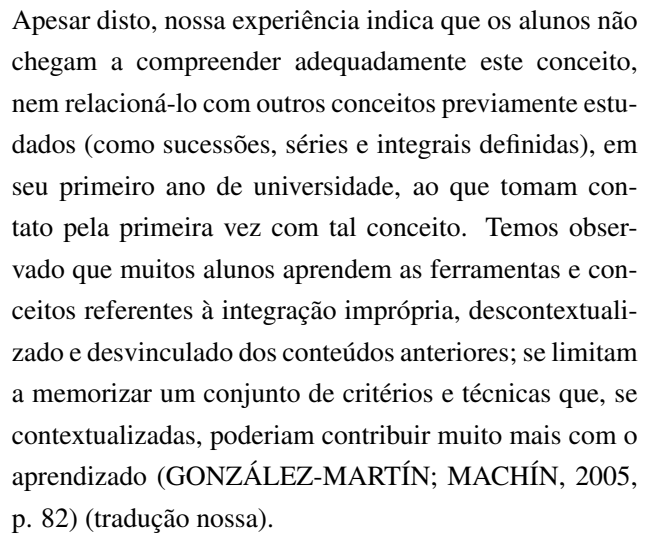

Apesar disto, nossa experiência indica que os alunos não chegam a compreender adequadamente este conceito, nem relacioná-lo com outros conceitos previamente estudados (como sucessões, séries e integrais definidas), em seu primeiro ano de universidade, ao que tomam contato pela primeira vez com tal conceito. Temos observado que muitos alunos aprendem as ferramentas e conceitos referentes à integração imprópria, descontextualizado e desvinculado dos conteúdos anteriores; se limitam a memorizar um conjunto de critérios e técnicas que, se contextualizadas, poderiam contribuir muito mais com o aprendizado (GONZÁLEZ-MARTÍN; MACHÍN 2005. p. 82) (tradução nossa).

González-Martín e Machín (2005, p. 82) (tradução nossa) evidenciam o caráter hegemônico de trabalhos que se desenvolvem a partir do marco teórico da integral definida. E, no contexto de integrais definidas, alertam que "nossos alunos não sabem quais as condições necessárias para se definir a integral de Riemann”. Ademais, acrescentam ainda as dificuldades inerentes em atribuir significado à integral como uma área, a ocorrência de falsas concepções, de modo que os alunos não diferenciam o sinal da integral quando a função é positiva, ou quando a função é negativa.

González-Martín e Machín (2005, p. 82) declaram empregar, como metodologia de pesquisa, uma Engenharia Didática, sobre o conceito de integral imprópria. Ademais, os dados discutidos por esses autores envolvem análises sobre a aprendizagem, conquanto que, não produziram implicações e análises do seu ensino. E, em relação a tal opção investigativa, acentuam que "neste aspecto, a descrição de um modelo de competência cognitiva, para caracterizar o nível de compreensão dos alunos e dos processos envolvidos, facilita um estudo qualitativo que se pretende realizar".

Nas suas recomendações finais, González-Martín e Machín (2005, p. 91) alertam para o fato de que, os alunos manifestam predileção por enunciados claros e diretos, com o apelo a uma abordagem algébrica. Os dados empíricos apontavam que "as perguntas de raciocínio e àquelas que requeriam interpretação, não foram abordadas satisfatoriamente, de modo geral". Ademais, os estudantes se mostram inseguros, quando se requisita uma formulação condizente com a definição formal de integral imprópria.

González-Martín e Machín (2005, p. 91) observam, segundo os dados das entrevistas, que os alunos não utilizam habitualmente gráficos como auxílio aos raciocínios mobilizados. Além de que, manifestam resis- 
VISUALIZAÇÃO DE INTEGRAIS DEPENDENTES DE PARÂMETROS COM ARRIMO NO SOFTWARE GEOGEBRA: UMA ENGENHARIA DIDÁTICA PARA SEU ENSINO

tências na explicação de estratégias envolvendo gráficos. Por outro lado, os autores sugerem fortemente que "uma correta compreensão do conceito de integral indefinida necessita visualizar o cálculo de áreas como um processo dinâmico, assim, tal visão permite conceber a função integral e avaliar seu limite" (GONZÁLEZMARTIN \& CAMACHO, 2005, p. 91, tradução nossa).

Em outro escrito científico, González-Martin \& Camacho (2004) descrevem as atividades desenvolvidas com um grupo de primeiro ano de estudos acadêmicos, no que concerne ao estudo de integrais impróprias. Tais autores se debruçam sobre a compreensão do fenômeno envolvendo o uso de registros gráficos em suas tarefas e de que modo os mesmos explicam-nas e agregam significado ao processo.

Logo no início, González-Martín e Camacho (2004 p. 479) declaram que "com a intenção de descrever um design de sequência de ensino, e desenvolver uma análise cognitiva dos dados oriundos das atividades de resolução dos estudantes, desenvolvemos uma investigação, contando com a participação de 25 estudantes”. Dessa forma, como característica das atividades estruturadas e abordadas com os aprendizes, eles assinalam ainda que "algumas das características das sequências de ensino dizem respeito à articulação entre os registros gráficos com o algébrico, a reconstrução do conhecimento a partir dos conceitos previamente estudados (séries de números reais e integrais definidas)".
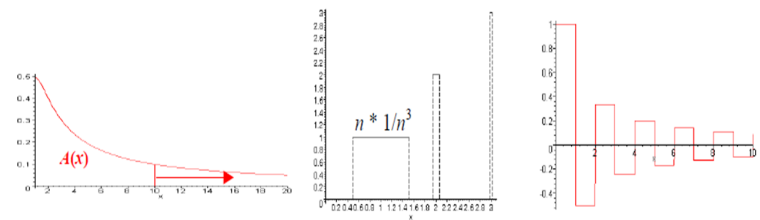

Figura 3: Registros gráficos relacionados à noção de integrais impróprias. Fonte: González-Martín e Camacho (2004 p. 481 - 482).

Na Figura 3 divisamos o empenho dos autores passados, no sentido de descrever/estruturar situações didáticas que envolvem menor recurso aos registros eminentemente algébricos. Com efeito, ao lado esquerdo da Figura 3, González-Martín e Camacho (2004, p. 481) observam que num primeiro momento é possível interpretar os gráficos e justificar a definição, envolvendo o uso de integrais impróprias. De modo específico, discutem as integrais:

$$
\begin{aligned}
& \int_{1}^{\infty} x^{-\frac{1}{3}} d x=\infty \\
& \int_{0}^{\infty} e^{-x} d x=1 .
\end{aligned}
$$

Por observarem que "as duas funções possuem gráfico bem similar, todavia, envolvem áreas bem diferentes".

Por sua vez, ao lado direito, analisam o comportamento da função $f(x)=\operatorname{sen}(x) / x$, cuja integral correspondente converge, todavia, não absolutamente. A função anterior, pode ficar à mercê da investigação intuitiva dos estudantes. Neste caso, GonzálezMartín e Camacho (2004, p. 482) comentam que "usando a teoria das séries e registros gráficos, se torna mais fácil a construção de registros gráficos envolvendo contra-exemplos de funções que convergem condicionalmente". No cenário indicado por esses autores, a visualização funciona de modo impulsionador de produção de conjecturas.

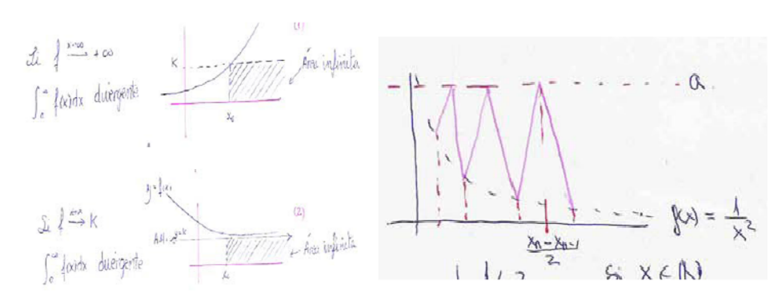

Figura 4: Produção dos alunos no contexto de ensino das integrais impróprias. Fonte: González-Martín e Camacho (2004 p. 482).

No que concerne ao cenário de produções que exibimos na Figura 4 González-Martín e Camacho (2004, p. 483) (tradução nossa) observam que "em nossa classe de observação, podemos claramente mencionar que os estudantes aceitam, gradualmente os registros gráficos, com a intenção de formular e conjecturar no momento de critérios de divergência". Um pouco mais adiante, comentam ainda que "depois, o trabalho é desenvolvido em grupos pequenos, e o professor fornece sua aprovação, o que funciona como a institucionalização de um registro, como um registro matemático". Na Figura 4 . divisamos uma profusão de registros gráficos produzidos pelos estudantes, todavia, nem todos se fundamentam num modelo confiável e que adquira status de matemático e possuidor de um rigor standard em Matemática.

\section{SOBRE O ENSINO DE INTEGRAIS DEPEN- DENTES DE PARÂMETROS}

Registramos poucos trabalhos que abordam algumas questões relacionadas com a noção de integrais dependentes de parâmetros, noção que generaliza o conceito de integral imprópria. Assim, nesta seção, assinalaremos e pormenorizaremos alguns elementos presentes em alguns artigos produzidos no Exterior (DANA- 

ENGENHARIA DIDÁTICA PARA SEU ENSINO

PICARD, 2005a; DANA-PICARD, 2005b; DANAPICARD, 2010, DANA-PICARD; ZEITOUN, 2012a, DANA-PICARD; ZEITOUN, 2012b). Registramos sua relevância, na medida em que, acentuam uma perspectiva unificadora, que envolve o conhecimento matemático que mobilizamos no estudo de integrais dependentes de parâmetros e sua convergência/divergência.

Na Figura 5 , Dana-Picard (2005b) acentua a perspectiva de que "o processo cognitivo ocorre quando a aprendizagem em Matemática não se restringe à composição de tópicos". Este autor cunha o termo cognitive neighborhood (vizinhança cognitiva), relativamente a determinado tópico matemático, como algo incluso num determinado espaço, que o mesmo nomeia de conhecimento matemático (mathematical knowledge). Os tópicos dispostos em uma determinada vizinhança são relacionados por conexões.

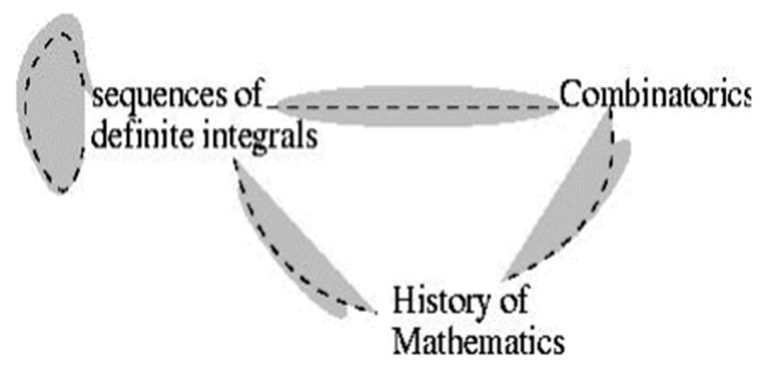

Figura 5: Relações conceituais e possibilidades da exploração didática das integrais definidas por parâmetros. Fonte: Dana-Picard 2005 b p. 7) - Sequences of definite integrals (Seqüência de integrais definidas); Combinatorics (Combinatoria); History of Mathematics (Historia da Matemática)

Por outro lado, na Figura 6 divisamos um ideograma que explica o significado do termo extended cognitive neighborhood (vizinhança cognitiva extendida). Em relação à ideia envolvida no esquema proposto por Dana-Picard (2005b, p. 7) (tradução nossa) esclarece que "não representa apenas conexões entre as noções e tópicos matemáticos, porém, envolve ainda instrumentos e os modos de seu uso". O nível inferior indica o conhecimento matemático. Enquanto que, o nível superior, indica técnicas e instrumentos que potencializam o significado do mesmo, para o sujeito que desenvolve uma ação investigativa.

Dana-Picard (2004, p. 1) (tradução nossa) alerta para o fato de que "num curso tradicional, quando um estudante fornece seu dever de casa, existem poucas chances de descobrir o quanto do trabalho foi elaborado seguindo um caminho puramente técnico". Neste sentido, Dana-Picard (2004) acentua o caso em que, a atividade do estudante se resume na aplicação de teore- mas e métodos de ensino vistos em sala de aula.

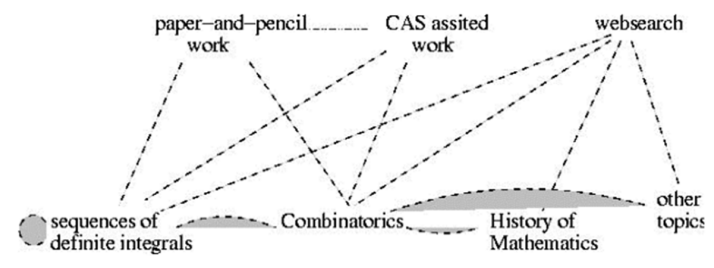

Figura 6: Diagrama que representa uma vizinhança cognitiva extendida (extended cognitive neighborhood), na concepção de DanaPicard. Fonte: Dana-Picard 2005b p. 8) - Sequences of definite integrals (Sequiência de integrais definidas); Combinatorics (Combinatória); History of Mathematics (História da Matemática); paper and- pencil (lápis e papel; Work (trabalho); websearch (pesquisa na internet); other topics (outros tópicos)

Outra alternativa, diz respeito ao empenho do estudante na direção de um profundo insight relativamente ao que foi designado como tarefa de casa. De modo geral, "os estudantes não tentam atribuir ao trabalho de casa um espectro de significação mais abrangente". Dana-Picard (2004, p. 1) (tradução nossa) acrescentar ainda "o valor agregado por intermédio de questões adequadas e observações elaboradas pelo educador" (DANA-PICARD, 2004, p. 1) (tradução nossa). Por tal via, a aprendizagem em Matemática pode se tornar mais compreensível, como advoga este autor.

Vejamos uma atividade proposta por Dana-Picard (2004, p. 1), no contexto do ensino de integrais dependentes de um parâmetro, com restrito de cunho analítico. Para tanto, o autor considera:

$$
I_{n}=\int_{0}^{a} x^{n} \sqrt{a^{2}-x^{2}} d x, \text { para } n \geq 0 .
$$

De imediato, conseguimos avaliar que:

$$
I_{0}=\int_{0}^{a} \sqrt{a^{2}-x^{2}} \text { e } I_{1}=\int_{0}^{a} x \sqrt{a^{2}-x^{2}} .
$$

Com efeito, vejamos que:

$$
\begin{gathered}
I_{0}=\int_{0}^{a} \sqrt{a^{2}-x^{2}} d x= \\
=\left[\frac{a^{2}}{2} \operatorname{arcsen}\left(\frac{x}{a}\right)+\frac{x}{2} \sqrt{a^{2}-x^{2}}\right]_{0}^{a}= \\
=\frac{1}{2} a^{2} \operatorname{arcsen}(1)=\frac{a^{2} \pi}{4}
\end{gathered}
$$

Conex. Ci. e Tecnol. Fortaleza/CE, v. 9, n. 4, p. 173 - 183, dez. 2015 
Enquanto que:

$$
\begin{aligned}
I_{1} & =\int_{0}^{a} x \sqrt{a^{2}-x^{2}} d x= \\
& =\left[-\frac{1}{3}\left(a^{2}-x^{2}\right)^{3 / 2}\right]_{0}^{a}= \\
& =\frac{a^{3}}{3} .
\end{aligned}
$$

No próximo passo, buscamos uma fórmula indutiva para a seqüência $\left(I_{n}\right)_{n \in \mathbb{N}}$. Dana-Picard (2004, p. 4) emprega integração por partes, ao escrever:

$$
\left\{\begin{array}{c}
u(x)=x^{n} \\
v(x)=\frac{1}{2} x \sqrt{a^{2}-x^{2}}+\frac{a^{2}}{2} \operatorname{arcsen}\left(\frac{x}{a}\right)
\end{array}\right.
$$

Daí, segue que:

$$
\begin{gathered}
I_{n}=\int_{0}^{a} x^{n} \sqrt{a^{2}-x^{2}} d x= \\
=\frac{1}{2} a^{n+2} \frac{\pi}{2}-\frac{n}{2} I_{n}-\frac{1}{2} a^{2} n \cdot \int_{0}^{a} x^{n-1} \operatorname{arcsen}\left(\frac{x}{a}\right) d x .
\end{gathered}
$$

Seja:

$$
K_{n}=\int_{0}^{a} x^{n-1} \operatorname{arcsen}\left(\frac{x}{a}\right) d x
$$

Assim:

$$
I_{n}=\frac{1}{2} a^{n+2} \cdot \frac{\pi}{2}-\frac{n}{2} I_{n}-\frac{1}{2} a^{2} n K_{n}
$$

Pode-se ainda escrever:

$$
\left(1+\frac{n}{2}\right) I_{n}=\frac{\pi}{4} a^{n+2}-\frac{1}{2} a^{2} n \cdot K_{n}
$$

Em seguida, Dana-Picard considera uma segunda integração por partes:

$$
\left\{\begin{array}{c}
u_{1}(x)=x^{n-1} \\
v_{1}(x)=\frac{1}{a}\left(x \cdot \operatorname{arcsen} \frac{x}{a}+\sqrt{a^{2}-x^{2}}\right)
\end{array}\right.
$$

Agora, considerando uma segunda integração por partes, tem-se: Sejam:

$$
\begin{gathered}
u_{1}=x^{n-1}=d u_{1}=(n-1) \cdot x^{n-2} d x \\
d v_{1}=\operatorname{arcsen}\left(\frac{x}{a}\right) d x \Rightarrow v_{1}=\int \operatorname{arcsen}\left(\frac{x}{a}\right) d x
\end{gathered}
$$

Resolvendo $\int \operatorname{arcsen}\left(\frac{x}{a}\right) d x$ obtém-se:

$$
\int \operatorname{arcsen}\left(\frac{x}{a}\right) d x=x \cdot \operatorname{arcsen}\left(\frac{x}{a}\right)+\sqrt{a^{2}-x^{2}}
$$

Assim tem-se que:

$$
\int_{0}^{a}\left(x^{n-1} \operatorname{arcsen}\left(\frac{x}{a}\right)\right) d x=
$$

Conex. Ci. e Tecnol. Fortaleza/CE, v. 9, n. 4, p. 173 - 183, dez. 2015 


$$
\begin{gathered}
=\left.x^{n-1} \cdot\left(x \cdot \operatorname{arcsen}\left(\frac{x}{a}\right)+\sqrt{a^{2}-x^{2}}\right)\right|_{0} ^{a}- \\
-\int_{0}^{a}\left(x \cdot \operatorname{arcsen}\left(\frac{x}{a}\right)+\sqrt{a^{2}-x^{2}}\right)(n-1) \cdot x^{n-2} d x \\
\int_{0}^{a}\left(x^{n-1} \operatorname{arcsen}\left(\frac{x}{a}\right)\right) d x= \\
=a^{n} \frac{\pi}{2}-\left[(n-1) \int_{0}^{a} x^{n-1} \operatorname{arcsen}\left(\frac{x}{a}\right) d x+(n-1) \int_{0}^{a}\left(x^{n-2} \sqrt{a^{2}-x^{2}}\right) d x\right] \\
\int_{0}^{a}\left(x^{n-1} \operatorname{arcsen}\left(\frac{x}{a}\right)\right) d x+(n-1) \int_{0}^{a}\left(x^{n-1} \operatorname{arcsen}\left(\frac{x}{a}\right)\right) d x=a^{n} \frac{\pi}{2}-(n-1) \int_{0}^{a}\left(x^{n-2} \sqrt{a^{2}-x^{2}}\right) d x \\
(1+n-1) \int_{0}^{a}\left(x^{n-1} \operatorname{arcsen}\left(\frac{x}{a}\right)\right) d x=a^{n} \frac{\pi}{2}-(n-1) \int_{0}^{a}\left(x^{n-2} \sqrt{a^{2}-x^{2}}\right) d x \\
n \int_{0}^{a}\left(x^{n-1} \operatorname{arcsen}\left(\frac{x}{a}\right)\right) d x=a^{n} \frac{\pi}{2}-(n-1) \underbrace{a}_{0}\left(x^{n-2} \sqrt{a^{2}-x^{2}}\right) d x \\
\int_{0}^{a}\left(x^{n-1} \operatorname{arcsen}\left(\frac{x}{a}\right)\right) d x=\frac{1}{n}\left(a^{n} \frac{\pi}{2}-(n-1) I_{n-2}\right)
\end{gathered}
$$

Assim:

$$
K_{n}=\int_{0}^{a}\left(x^{n-1} \operatorname{arcsen}\left(\frac{x}{a}\right)\right) d x=\frac{1}{n}\left(a^{n} \frac{\pi}{2}-(n-1) I_{n-2}\right)
$$

Já foi visto anteriormente que:

Substituindo $K_{n}$

$$
\left(1+\frac{n}{2}\right) I_{n}=\frac{\pi}{4} a^{n+2}-\frac{1}{2} a^{2} n K_{n}
$$

$$
\begin{gathered}
\left(1+\frac{n}{2}\right) I_{n}=\frac{\pi}{4} a^{n+2}-\frac{1}{2} a^{2} n \frac{1}{n}\left(a^{n} \frac{\pi}{2}-(n-1) I_{n-2}\right) \\
\left(1+\frac{n}{2}\right) I_{n}=\frac{\pi}{4} a^{n+2}-\frac{\pi}{4} a^{n+2}+\frac{a^{2}(n-1)}{2} I_{n-2},
\end{gathered}
$$

Logo:

$$
I_{n}=\frac{a^{2}(n-1)}{n+2} I_{n-2}
$$

Nas próximas etapas, Dana-Picard (2004) acentua sua interpretação e, logo em seguida, atividades assistidas por computador que permitem a exploração do modelo discutido há pouco. Dana-Picard (2005b, p. 4) acentua o seguinte modelo de recorrência

$$
I_{n}=f(n) \cdot I_{n-1},
$$

onde indica uma homografia, na variável, com coeficientes inteiros. Dana-Picard (2005b, p. 4 - 5) (tradução nossa) adverte que "quando esta situação não ocorre, os cálculos podem se tornar mais complicados". 
VISUALIZAÇÃO DE INTEGRAIS DEPENDENTES DE PARÂMETROS COM ARRIMO NO SOFTWARE GEOGEBRA: UMA ENGENHARIA DIDÁTICA PARA SEU ENSINO

\section{POSSIBILIDADES DO ENSINO DE INTE- GRAIS DEPENDENTES DE PARÂMETROS COM ARRIMO NO SOFTWARE GEOGEBRA}

Na seção anterior abordamos uma perspectiva de ensino acentuada por Dana-Picard, ao desenvolver um estudo e proposta, tendo em vista o ensino e a aprendizagem da noção de integrais dependentes de parâmetros. Nessa seção acentuaremos as potencialidades do uso do software GeoGebra tendo em vista a visualização de propriedades e relações que podem ser ressignificadas, na medida em que apoiamos nossa mediação na tecnologia atual.

Na Figura 7, temos a possibilidade de explorar o comportamento de convergência ou divergência da integral descrita por:

$$
I_{n}=\int_{0}^{+\infty} \frac{1}{\left(x+\sqrt{1+x^{2}}\right)^{n}} d x
$$

ao lado esquerdo. Com origem em uma manipulação do estudante de seletores móveis do GeoGegra, os alunos poderão depreender que as contribuições dinâmicas de área (de cor amarela) tendem paulatinamente a decrescer, na medida em que $x \rightarrow+\infty$. Ora, ao lado direito, com origem no exemplo discutido na seção anterior, sabemos que:

$$
I_{n}=\frac{n}{n^{2}-1}
$$

E, com origem no rastro (na cor azul) definido no plano cartesiano por $\left(n, \frac{n}{n^{2}-1}\right) \in \mathbb{R}^{2}$, os estudantes poderão agora concluir que seus valores não assumem valores demasiadamente grandes, na medida em que $x \rightarrow+\infty$. Entretanto, podemos analisar, de modo particular, os valores iniciais de seu comportamento.

Todavia, cabe observar que:

$$
I_{1}=\int_{0}^{+\infty} \frac{1}{\left(x+\sqrt{1+x^{2}}\right)} d x
$$

E usando que:

$$
x=\operatorname{senh}(t) \therefore d x=\cosh (t)
$$

Podemos substituir:

$$
\begin{aligned}
& I_{1}=\int_{0}^{+\infty} \frac{1}{\left(x+\sqrt{1+x^{2}}\right)} d x= \\
& =I_{1}=\int_{0}^{+\infty} \frac{\cosh (t)}{\operatorname{senh}(t)+\cosh (t)} \\
& \begin{array}{l}
\cosh (t)=\frac{e^{t}+e^{-t}}{2} \\
\operatorname{senh}(t)=\frac{e^{t}-e^{-t}}{2}
\end{array} \int_{0}^{+\infty} \frac{e^{t}+e^{-t}}{2 e^{t}} d t=
\end{aligned}
$$

Conex. Ci. e Tecnol. Fortaleza/CE, v. 9, n. 4, p. 173 - 183, dez. 2015

$$
=\int_{0}^{+\infty}\left(1+e^{-2 t}\right) d t=\frac{1}{2}\left(t-\frac{e^{-2 t}}{2}\right)=+\infty
$$

Portanto, divergente para $n=1$. Desse modo, com origem na visualização, os alunos podem fazer os devidos ajustes do modelo indutivo, e considerar com comportamento de convergência da integral

$$
I_{1}=\int_{0}^{+\infty} \frac{1}{\left(x+\sqrt{1+x^{2}}\right)^{n}} d x=
$$

, com a condição de $n \geq 2$.

Por outro lado, na Figura 8 poderemos conduzir os estudantes ao entendimento da divergência da referida integral, na medida em que, os mesmo podem visualizar ao lado esquerdo, as contribuições tendencialmente crescentes (na cor amarela), oriundas do valor numérico da integral anterior. Ao lado direito, o comportamento esperado dos pontos do tipo $\left(n, \frac{n}{n^{2}-1}\right) \in \mathbb{R}^{2}$, devem ser do tipo de afastamento do eixo das abcissas. Desse modo, a partir da "soma visual" envolvendo a ação perceptual de coligir os dados exibidos nas duas janelas do GeoGebra, os alunos poderão depreender o comportamento de divergência, quando exploramos os índices $I_{n}, \operatorname{com} n>0$.

Finalmente, com a influência da perspectiva sugerida por Dana-Picard, e da noção de vizinhança cognitiva extendida, temos condições de relacionar dados analíticos de resoluções de integrais, como dados qualitativos visuais fornecidos pelo modelo computacional, com ênfase nas relações conceituais pretendidas por Dana-Picard.

\section{CONSIDERAÇÕES FINAIS}

Nas seções anteriores traçamos um cenário de estudo, atinente as investigações realizadas com o tema Integrais Impróprias ou Integrais Generalizadas. Do ponto de vista matemático, a noção de integrais dependentes de parâmetros permite uma via de generalização do referido modelo e, desse modo, perspectivamos uma significação didático-metodológica caracterizada por Dana-Picard (2004), Dana-Picard (2005a) e DanaPicard (2005b).

Dessa forma, diante de entraves persistentes e manifestos por estudantes, que possuem raízes desde a aprendizagem da noção de integrais definidas, no contexto de aprendizagem do Teorema Fundamental do Cálculo, e persistem até o estudo da noção de integrais dependentes de parâmetros. Recordamos a tese de Alves (2011), em que nomeia o processo de Transição Interna do Cálculo, relacionada aos processos de avaliação de limite, derivação e integração. Notamos que, no 
VISUALIZAÇÃO DE INTEGRAIS DEPENDENTES DE PARÂMETROS COM ARRIMO NO SOFTWARE GEOGEBRA: UMA ENGENHARIA DIDÁTICA PARA SEU ENSINO

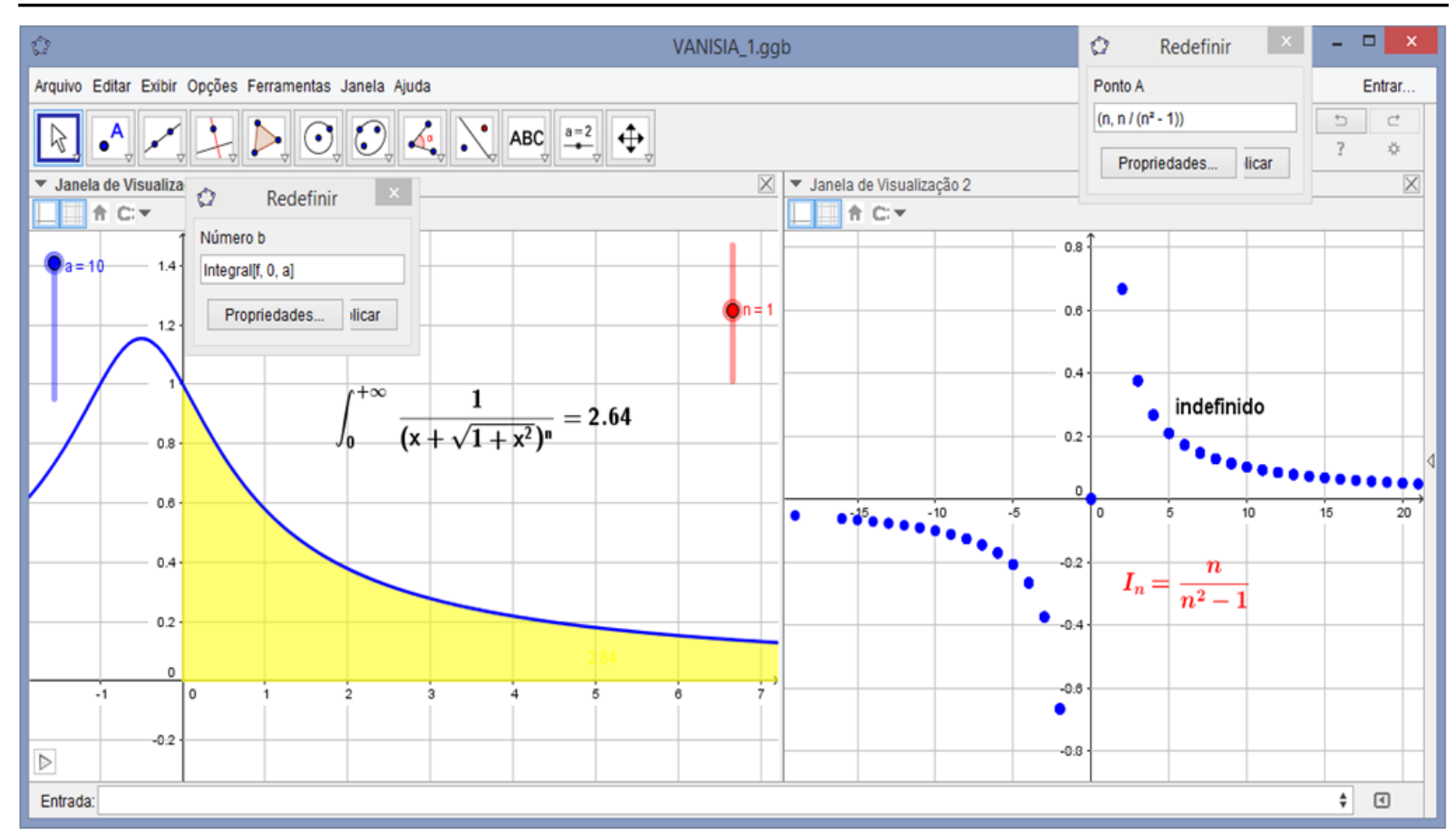

Figura 7: Soma visual relativa a uma vizinhança cognitiva relacionada com a noção de integrais dependentes de parâmetros no caso da convergência. Fonte: Autoria própria.

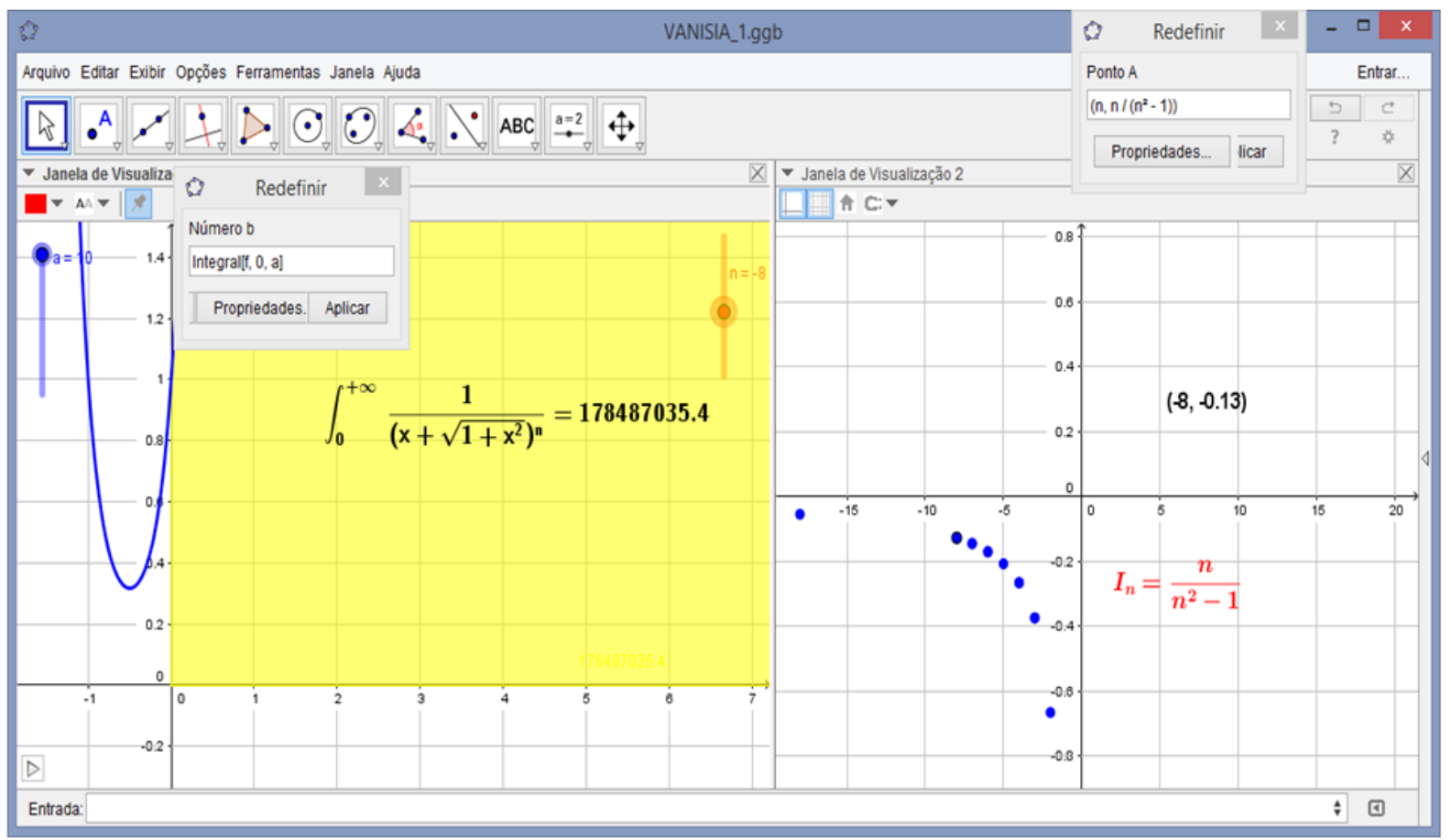

Figura 8: Soma visual relativa a uma vizinhança cognitiva relacionada com a noção de integrais dependentes de parâmetros no caso da divergência. Fonte: Autoria própria. 
VISUALIZAÇÃO DE INTEGRAIS DEPENDENTES DE PARÂMETROS COM ARRIMO NO SOFTWARE GEOGEBRA: UMA ENGENHARIA DIDÁTICA PARA SEU ENSINO

caso que discutimos aqui, pode ser enquadrado no caso da Transição do processo de integração, como divisamos na Figura 9

$$
\int_{\substack{a \\ \text { INIEGRAIS } \\ D E F I N I D A S}}^{b} f(x) d x \Rightarrow \int_{\substack{a \\ \text { GNTEGRAIS } \\ \text { GENERALIZADAS }}}^{+\infty} f(x) d x \Rightarrow \int_{\substack{a \\ I N I E G R A I S \\ D E P E N D E N I E S \\ D E \text { PARAMETROS }}}^{+\infty} f_{n}(x) d x
$$

Figura 9: Transição Interna do Cálculo no caso do processo de integração. Fonte: Autoria própria.

Para concluir, depreendemos que, a ação dos estudantes desconsiderando a tecnologia atual não permite a exploração de um espectro de possibilidades sugeridas, por exemplo, pelo software GeoGebra, empregado no último exemplo. No que concerne ao papel do professor e do papel do estudante, não exigimos grande domínio de um sintaxe programacional, posto que, o software possui cultura acadêmica razoavelmente conhecida pela sua simplicidade e exploração dinâmica dos conceitos científicos matemáticos.

Enfim, os dados abordados e coligidos no presente artigo fornecem os indicadores preliminares para nossa investigação de mestrado em Ensino de Ciências e Matemática. Em etapas ulteriores, perspectivamos o emprego do ponto de vista de Dana-Picard com o escopo de formular/estruturar situações problemas, ancoradas na ideia de vizinhança cognitiva extendida, tendo em vista a superação dos obstáculos indicados preliminarmente.

\section{REFERÊNCIAS}

ALVES, F. R. V. Aplicações da Sequência Fedathi na promoção das categorias do raciocínio intuitivo no Cálculo a Várias Variáveis. Tese (Programa de Pós-graduação em Educação Brasileira) Universidade Federal do Ceará (UFC), Fortaleza, 2011. $399 \mathrm{f}$.

ALVES, F. R. V. Transição interna do cálculo: uma discussão do uso do geogebra no contexto do cálculo a várias variáveis. Revista do Instituto GeoGebra Internacional de São Paulo. ISSN 2237-9657, v. 1, n. 2, p. 5-19, 2012. Disponível em: <http://revistas.pucsp. br/index.php/IGISP/article/view/11373>.

Visual criterion for understanding the notion of convergence if integrals in one parameter. Acta Didactica Napocensia, Babes Bolyai University, Didactics of Exact Sciences Chair, v. 7, n. 1, p. 19, 2014. Disponível em: <http://search.proquest.com/ openview/ba5f19c1ad6481c19df32b73a8a64533/1. pdf?pq-origsite $=$ gscholar \&cbl=2028913>

Visualização de integrais impróprias em um parâmetro com o auxílio do geogebra. \# Tear: Revista de Educação, Ciência e Tecnologia, v. 3, n. 1, 2014. Disponível em: <http://seer.canoas.ifrs.edu.br/seer/ index.php/tear/issue/view/5/showToc>

ALVES, F. R. V.; LOPES, M. A. Métodos de integração: uma discussão do seu ensino com apoio no software geogebra. Revista do Instituto GeoGebra Internacional de São Paulo. ISSN 2237-9657, v. 2, n. 1, p. 05-21, 2013. Disponível em: <http://revistas.pucsp. br/index.php/IGISP/article/view/12524>

BERMÚDEZ, E. A. Comprensión del concepto de integral definida en el marco de la teoría APOE. Tese (Doutorado) - Universidad de Salamanca, Faculdade de lãs Ciencias Experimentales, Salamanca, 2011. $423 f$.

DANA-PICARD, T. Explicit closed forms for parametric integrals. International Journal of Mathematical Education in Science and Technology, Taylor \& Francis, v. 35, n. 3, p. 456-467, 2004.

Parametric integrals and catalan numbers. International Journal of Mathematical Education in Science and Technology, Taylor \& Francis, v. 36, n. 4, p. 410-414, 2005.

Sequences of definite integrals, factorials and double factorials. Journal of Integer Sequences, v. 8, n. 2, p. 3, 2005. Disponível em: <https://cs.uwaterloo. ca/journals/JIS/VOL8/Dana-Picard/dana23.pdf>

Technology-assisted discovery of conceptual connections within the cognitive neighborhood of a mathematichal topic. In: Proceedings of CERME 4. 2005. p. 1 - 9. Disponível em: $<$ http://fractus.uson.mx/Papers/CERME4/Papers\% 20definitius/9/Dana-Picard.pdf>

Integral presentations of catalan numbers. International Journal of Mathematical Education in Science and Technology, Taylor \& Francis, v. 41, n. 1, p. 63-69, 2010. Disponível em: $<$ http://www.tandfonline.com/doi/pdf/10.1080/ 00207390902971973>

DANA-PICARD, T.; ZEITOUN, D. G. Parametric improper integrals, wallis formula and catalan numbers. International Journal of Mathematical Education in Science and Technology, Taylor \& Francis, v. 43, n. 4, p. 515-520, 2012. Disponível 
VISUALIZAÇÃO DE INTEGRAIS DEPENDENTES DE PARÂMETROS COM ARRIMO NO SOFTWARE GEOGEBRA: UMA ENGENHARIA DIDÁTICA PARA SEU ENSINO

em: <http://www.tandfonline.com/doi/pdf/10.1080/ 0020739X.2011.599877>

Sequences of definite integrals, infinite series and stirling numbers. International Journal of Mathematical Education in Science and Technology, Taylor \& Francis, v. 43, n. 2, p. 219-230, 2012. Disponível em: <http://www.tandfonline.com/doi/abs/ 10.1080/0020739X.2011.582172>

GONZÁLEZ-MARTÍN, A. S. La generalización de la integral definida desde las perspectivas numérica, gráfica y simbólica utilizando entornos informáticos: problemas de enseñanza y aprendizaje. Tese (Doutorado) — Universidad de La Laguna, Laguna, 2005a. 498f.

GONZÁLEZ-MARTÍN, A. S. The use of technology and mathematical results in parallel a case with improper integration. In: Proceeedings of International Comission of Studyand Improvment of Mathematics Teaching - CIEAEM/57. Itália: , 2005b. p. 165 - 169. Disponível em: <http://math.unipa.it/ grim/cieaem/ cieaem57_gonzales_martin.pdf $>$

GONZÁLEZ-MARTÍN, A. S.; CAMACHO, M. What is first-year mathematics students' actual knowledge about improper integrals? International journal of mathematical education in science and technology, Taylor \& Francis, v. 35, n. 1, p. 73-89, 2004.

GONZÁLEZ-MARTÍN, A. S.; MACHÍN, M. C. Sobre la comprensión en estudiantes de matemáticas del concepto de integral impropia. Enseñanza de las Ciencias, v. 23, n. 1, p. 081-96, 2005.

HSIA, Y. W. A utilização do livro didático por parte do aluno ao estudar integral. Dissertação (mestrado em Educação Matemática) - Pontifícia Universidade de São Paulo, São Paulo, 2006. 87f. Disponível em: <http://www.sapientia.pucsp.br/tde_arquivos/3/ TDE-2007-05-08T09:50:22Z-3063/Publico/EDM\% 20-\%20Yuk\%20Wah\%20Hsia.pdf>.

MILOVANOVIĆ, M.; TAKAČI, Đ.; MILAJIĆ, A. Multimedia approach in teaching mathematicsexample of lesson about the definite integral application for determining an area. International Journal of Mathematical Education in Science and Technology, Taylor \& Francis, v. 42, n. 2, p. 175-187, 2011. Disponível em: <http://www.tandfonline.com/ doi/pdf/10.1080/0020739X.2010.519800>.

SEVIMLI, E.; DELICE, A. The influence of teacher candidates' spatial visualization ability on the use of multiple representations in problem solving of definite integrals: a qualitative analysis. Research in Mathematics Education, Taylor \& Francis, v. 13, n. 1, p. 93-94, 2011. Disponível em: <http://www.tandfonline.com/doi/pdf/10.1080/ 14794802.2011.550750>

The relationship between students' mathematical thinking types and representation preferences in definite integral problems. Research in Mathematics Education, Taylor \& Francis, v. 14, n. 3, p. 295-296, 2012.

TOMÉ, M. T. Integral definida, cálculo mental y nuevas tecnologias. Tese (Doutorado) - Universidad de Valladolid, Valladolid, 2011. 1114f. Disponível em: <http://www.tesisenred.net/handle/10803/80932>.

VANINSKY, A. Integral definition of the logarithmic function and the derivative of the exponential function in calculus. International Journal of Mathematical Education in Science and Technology, Taylor \& Francis, v. 46, n. 3, p. 450-456, 2015. 